\title{
La planification linguistique française : traditions et impact de la Communauté européenne
}

Anne Judge

\section{OpenEdition}

1 Journals

Édition électronique

URL : http://journals.openedition.org/ries/4222

DOI : 10.4000/ries.4222

ISSN : 2261-4265

Éditeur

Centre international d'études pédagogiques

Édition imprimée

Date de publication : 1 septembre 1994

Pagination : 33-46

ISSN : 1254-4590

\section{Référence électronique}

Anne Judge, «La planification linguistique française : traditions et impact de la Communauté européenne », Revue internationale d'éducation de Sèvres [En ligne], 03 | 1994, mis en ligne le 17 avril 2015, consulté le 01 mai 2019. URL : http://journals.openedition.org/ries/4222 ; DOI : 10.4000/ ries. 4222

Ce document a été généré automatiquement le 1 mai 2019.

(c) Tous droits réservés 


\title{
La planification linguistique française: traditions et impact de la Communauté européenne
}

\author{
Anne Judge
}

1 En France, la langue a toujours été affaire d'État. C'est encore le cas aujourd'hui : citons à ce sujet un passage tiré de l'exposé des motifs à l'origine du projet de loi relatif à l'emploi de la langue française, présenté récemment au Sénat et à l'Assemblée nationale :

Depuis l'ordonnance de Villers-Cotterêts de 1539 - qui dispose que la justice est rendue en français - et la création de l'Académie française en 1635 - qui a donné à notre langue un gardien - la langue française, ciment de l'unité nationale et élément fondamental de notre patrimoine, a fait l'objet de politiques publiques.

Que la langue joue un rôle primordial dans la conception de la nation française semble être vrai pour la majorité des Français si l'on en croit le sondage réalisé par la SOFRES pour le ministère de la culture et de la francophonie en mars $1994^{1}$ : $97 \%$ des Français seraient attachés à leur langue, et $70 \%$ fiers de sa diffusion internationale. Que la langue soit du domaine de la politique gouvernementale semble également vrai pour la majorité des personnes interrogées: pour $39 \%$, la défense de la langue française doit être un objectif très important pour le gouvernement, pour $51 \%$, il s'agirait d'un objectif important mais sans plus. Seuls $9 \%$ penseraient qu'il s'agit là d'un objectif peu important. Ces chiffres semblent légitimer une forte politique linguistique qui est, de fait, traditionnelle en France, quelle que soit la tendance des gouvernements.

Voilà de quoi surprendre un étranger : en Grande-Bretagne, par exemple, il n'y a tout simplement pas de législation linguistique, le développement de la langue étant canalisé uniquement par des forces sociales. Ceci n'empêche pas d'ailleurs que les Français interrogés lors du sondage mentionné ci-dessus aient déclaré que l'Angleterre faisait plus pour la défense de sa langue que la France, ce qui n'est pertinemment pas le cas. Ils semblent penser qu'une politique en matière linguistique est tout ce qu'il y a de plus normal. 
4 Le fait d'avoir une politique visant à défendre et à promouvoir le français a donc été jusqu'à tout récemment un sujet de consensus politique national. Cependant des failles sont apparues dans cet édifice de lois et d'institutions vieux d'un demi-millénaire lors des discussions récentes sur le projet de loi Toubon. Celui-ci ne contient rien, en fait, de bien nouveau mais vient simplement renforcer la loi Bas-Lauriol de 1975 (voir plus bas) qui vise à protéger les Français contre l'emploi de langues autres que le français dans les services publics, dans les entreprises, dans les médias, dans les campagnes publicitaires et surtout dans l'étiquetage des produits vendus dans le commerce. De plus, d'après la nouvelle loi, la présence du français serait renforcée lors de colloques et congrès. En cas d'infractions, les sanctions seront aggravées. Mais, alors que la loi Bas-Lauriol fut votée à l'unanimité en 1975 , la loi Toubon a été l'objet de discussions très animées, surtout à l'Assemblée nationale. Comme ce débat bouleverse les clivages politiques traditionnels, on peut se demander justement si certains Français ne commencent pas à s'interroger sur le bien-fondé de la politique linguistique traditionnelle.

5 Il existerait, de plus, à l'heure actuelle, des malentendus entre le gouvernement, les Français et les commentateurs dans les médias, en ce que le gouvernement et les Français ne confondraient pas politique de défense de la langue et chasse aux mots étrangers, ce qui est souvent le cas des commentateurs qui ont été très ironiques au sujet de la nouvelle loi Toubon. C'est du moins ce que dit le communiqué du 8 mars 1994 issu du ministère de la culture et de la francophonie ; il cite en particulier le fait que le sondage de la SOFRES montre que la majorité des Français approuve l'utilisation de mots étrangers, la trouve utile $(30 \%)$, amusante $(19 \%)$ et surtout moderne ( $41 \%)$. Seuls $16 \%$ trouvent que ce procédé fait snob, $3 \%$ choquant et $6 \%$ abêtissant. Les Français veulent donc dans leur ensemble que leur langue puisse s'enrichir d'emprunts étrangers. Le gouvernement affirme dans ce communiqué adopter exactement la même approche. Or le projet de loi Toubon a été présenté dans presque toute la presse comme représentant une politique purement défensive ( C'est la coïncidence qui tue : la France va adopter une loi tentant d'ériger des barbelés autour de sa langue à la veille de l'ouverture d'un tunnel censé nous rapprocher de nos voisins anglais » lisait-on dans Info-Matin le 5 mai 1994, à l'issue du débat du projet à l'Assemblée). Mais serait-ce possible que le malentendu soit non pas le résultat d'un désir de désinformation mais plutôt le signe d'un changement d'attitude de la part des journalistes quant à la légitimité d'une telle législation? Dans ce cas les médias représenteraient une avant-garde. En effet, comme nous le verrons plus bas, il y a à l'heure actuelle des voix qui s'élèvent contre le principe même de la légitimité de toute intervention législative dans le domaine de la langue au nom de la liberté d'expression. Notons également que le sondage de la SOFRES ne prend pas en considération les jeunes ayant moins de 18 ans, qui se sentent peut-être moins engagés par cette question que leurs aînés.

6 Pour voir plus clair dans ce domaine, examinons de manière plus détaillée l'évolution historique de la politique linguistique française et l'impact de la mise en place d'une Communauté européenne dont les règles linguistiques demeurent relativement floues.

7 La planification linguistique française traditionnelle a varié selon le stade auquel en était la langue: langue du roi au $\mathrm{X}^{\mathrm{e}}$ siècle, langue littéraire $d u \mathrm{XII}^{\mathrm{e}}$ au $\mathrm{XV}^{\mathrm{e}}$ siècle, langue administrative au XVI ${ }^{e}$ siècle, et langue de l'élite européenne au XVIII siècle, situation qui s'est prolongée en Russie jusqu'à la Révolution et en Pologne jusqu'à la deuxième guerre mondiale. Il ne s'agissait pas de la langue du peuple bien sûr, mais de la langue de l'intelligentsia, c'est-à-dire d'une langue travaillée par les écrivains et les intellectuels 
dans les salons et académies. C'est dans le contexte de cette évolution qu'est né le concept du français « langue pure, langue claire, langue parfaite».

Il faut remarquer tout d'abord qu'à l'origine de la planification linguistique française se trouvent des problèmes d'ordre pratique (manque de clercs sachant le latin et, surtout lors des famines, épidémies et guerres, besoin de textes juridiques et administratifs écrits en une langue compréhensible pour les personnes concernées, etc.). Ces problèmes ont eu pour conséquence l'apparition de textes juridiques et administratifs en français, là où la tradition exigeait qu'ils soient en latin. La question linguistique apparait pour la première fois dans un texte juridique en 1490 dans les Ordonnances de Moulins proclamées par Charles VIII, qui établissent que «les dits et dépositions de témoins dans les cours de justice du Languedoc seront mis ou rédigés en langage français ou maternel, tels que les dits témoins puissent entendre leurs dépositions et on les leur puisse dire et recenser en tel langage et forme qu'ils auront dit et déposé». Le but de cette loi était donc d'ordre pratique et pour le bien de tous.

Cependant la date la plus célèbre est 1539, date de la proclamation de l'édit de VillersCotterêts qui a fait du français - à l'exclusion de toute autre langue - la langue de l'administration et de la justice. Dès ce moment-là, les langues régionales perdront rapidement du terrain par rapport au français dans tout ce qui était écrit. Le peuple, cependant, a continué à utiliser les langues régionales dans la communication orale pendant près de quatre siècles. Notons que les motifs en arrière-plan de cette ordonnance n'étaient pas seulement d'ordre linguistique. François $\mathrm{I}^{\mathrm{er}}$, ne parlant pas le latin, se sentait désavantagé par rapport à ses clercs, et l'imposition du français dans les domaines administratif et juridique aidait à l'unification et à la centralisation du royaume. Dans ce cas la nouvelle législation constituait un outil politique.

10 La première institution ayant été créée pour planifier le développement du français est l'Académie française, fondée en 1635, qui marque le début de la standardisation officielle $\mathrm{du}$ français et du règne de Vaugelas. Le point de départ était raisonnable. Il s'agissait d'affirmer que le français était potentiellement une aussi bonne langue que le latin et de l'aider à devenir une langue aussi cohérente que possible. Tout le monde s'y est mis : grammairiens, écrivains, philosophes. Le résultat est que, petit à petit, l'idée s'est répandue que le français était la langue la plus claire, la plus logique et la plus précise du monde, puisque la France était le seul pays où des efforts conscients et systématiques aient été faits dans ce sens à tous les niveaux et dans tous les domaines; en raison de sa précision et de sa clarté, c'était la langue idéale de la diplomatie internationale ; c'était également une langue pure, descendant directement (ou presque) du latin et non une langue hybride comme l'anglais.

11 Une des conséquences de ces croyances : la nécessité de défendre la langue française. Ce principe remonte, en fait, à une période antérieure à celle de la fondation de l'Académie : son publiciste le plus célèbre est Du Bellay, auteur d'une Défense et illustration de la langue française publiée en 1549. Ce point de vue est rapidement devenu une constante de la vie française qui a survécu jusqu'à aujourd'hui. Le désir de défendre la langue s'accompagne tout naturellement de la croyance en un français idéal auquel l'humble mortel ne peut qu'aspirer. Cette croyance peut paraître ridicule vue de l'étranger. C'est ainsi que le Guardian du 26 avril 1994 reproduit un article du Monde intitulé « Parleying in French » de Bertrand Poirot-Delpech, membre de l'Académie française, qui affirme, pour la joie des lecteurs anglais, que pour sauver le français il faudrait que tous apprennent par cœur un 
bon livre de grammaire. Dans ce même article, Bertrand Poirot-Delpech revient, d'une manière caractéristique, aux sources quand il affirme :

The defence of the spirit of a language is impossible without what Du Bellay referred to as illustration or glorification. Through glorification, a kingdom may reach full splendour. Glorification means giving preference to the French word when the imported word adds nothing.

12 Le principe de «l'illustration" ou "glorification» de la langue française peut sembler ridicule - surtout en traduction anglaise - mais lorsque les scientifiques disent aujourd'hui qu'il faut que le gouvernement donne plus d'argent pour faire des recherches qui attireront les étrangers dans les laboratoires français ou que les cadres en mercatique (ou marketing!) disent que seules des idées ou inventions originales aideront à imposer le français à l'étranger, il s'agit bien d'illustration ou de glorification de la langue. En d'autres termes, et pour citer toujours Bertrand Poirot-Delpech, c'est seulement lorsque la France aura retrouvé sa puissance industrielle que le vocabulaire suivra. Notons donc qu'il ne s'agit pas seulement de défendre la langue pour elle-même, mais parce qu'elle est à la fois source et symbole de puissance politique et économique à l'échelle internationale. Or la "dégradation" du français est vue à l'heure actuelle comme reflétant sa perte de prestige dans le monde et la France étant essentiellement un pays nationaliste, elle a du mal à accepter cet état de fait.

Cependant dans un rapport présenté à l'Assemblée nationale au nom de la Commission des affaires culturelles, familiales et sociales sur le projet de loi Toubon, le 21 avril 1994, le député Francisque Perrut présente une nouvelle approche puisqu'il se déclare partisan de prendre Rabelais plutôt que Vaugelas comme modèle pour l'enrichissement de la langue. Certes, il proclame de manière très orthodoxe que « le français, de tradition latine et cartésienne, aime la précision, la nuance, les contours clairs du concept. Il pousse par son vocabulaire et sa syntaxe à un net compartimentage des notions et à une appréhension que l'on pourrait qualifier de rationalisant de la vie, de la pensée et des cultures ». Mais, et c'est là une attitude nouvelle, il se déclare du même avis que l'Acadienne Antonine Maillet quand elle affirmait, en parlant de la langue française importée au Canada :

Nous avons hérité de la langue qui n'avait pas encore été purifiée mais aussi appauvrie par l'Académie [...] Le mot n'est pas seulement communication, il est aussi expression sensuelle ou émotive de l'intelligence; si dans Rabelais il y a cent mille mots, c'est qu'il y a cent mille manières de dire une chose.

Et Francisque Perrut de conclure qu'il faut revenir aux méthodes de renouvellement chères à Rabelais, en l'occurrence aux emprunts aux cultures régionales et, par extension, aux créations des pays francophones. Il cite également le sociologue Jules Gritti à ce sujet: "Oublier quelque peu Voltaire ou Vaugelas pour retrouver le chanoine Rabelais [est] une condition pour un français ressourcé et savoureux ».

Pour en revenir à la mise en place du français en tant que langue nationale, VillersCotterêts ayant fait du français la langue de la justice, et l'Académie ayant été créée comme gardienne de la langue, c'est la Révolution française qui fournira la grande étape suivante. En effet le premier obstacle qu'aient eu à surmonter les révolutionnaires était l'absence d'une langue commune par laquelle faire connaître le message révolutionnaire. Leur premier acte en matière linguistique a été d'organiser, sous la direction de l'abbé Grégoire, la mise en place du premier questionnaire linguistique en 1790. Il était bien connu que le français était parlé par certaines élites à l'étranger depuis fort longtemps, mais qu'en était-il du peuple français ? Que parlait-il ? Le rapport Grégoire démontra qu'il 
parlait des langues dites régionales. Ceci a conduit à la décision gouvernementale de supprimer les langues et dialectes régionaux au nom de l'égalité des chances. Il y a eu beaucoup trop de résistance à cette politique pour qu'on puisse parler de consensus, mais il y a eu consensus au niveau des gouvernements, puisqu'ils ont tous travaillé dans ce but. Il est vrai que dans le nouveau projet de loi Toubon, nous lisons à l'article 19: «Les dispositions de la présente loi s'appliquent sans préjudice de la législation et de la réglementation relatives aux langues régionales ». Mais c'est là trop peu et trop tard pour qu'elles puissent être sauvées: on ne les combat plus, uniquement parce qu'elles ne représentent plus aucun danger.

16 Ayant décidé qu'il était nécessaire d'imposer le français comme langue nationale, Talleyrand déposa un rapport à la Constituante en 1791 demandant l'établissement d'écoles primaires dans lesquelles serait enseigné le français. Il était clair dès ce momentlà que l'enseignement $d u$ français en serait un des piliers. Mais les révolutionnaires ont été vite dépassés par les événements. Il a fallu attendre 1832 pour que le gouvernement mette en place un système d'éducation primaire et décide d'une orthographe officielle, celle du Dictionnaire de l'Académie, obligatoire pour tous les examens et dans tous les documents officiels; ensuite les lois de Jules Ferry en 1885 pour que l'enseignement devienne obligatoire; et enfin la première guerre mondiale pour que s'opère le grand brassage des populations qui a fait progresser de manière spectaculaire la réalité d'un français, langue de tous en France. Ceci est chose faite depuis la seconde guerre mondiale en ce sens que tous les enfants nés en France connaissent le français, mis à part, bien entendu, les enfants d'étrangers ne parlant que leur langue d'origine à la maison. Ceci dit, même ces enfants-là apprendront le français à l'école et la génération suivante aura le français comme seule langue maternelle ${ }^{2}$.

17 Autrefois donc une des missions de l'école était d'imposer le français comme seule langue nationale. C'est chose faite. La nouvelle mission de l'école, d'après de nombreux articles publiés dans le contexte de la discussion au sujet de la loi Toubon, est de réintroduire chez les jeunes l'emploi d'un bon français (concept qui ne fait cependant pas l'unanimité). C'est ainsi que nous lisons dans le rapport fait au nom de la commission des affaires culturelles, familiales et sociales cité ci-dessus : «La défense de la langue française passe avant tout par son enseignement: or, la plupart des enfants entrent aujourd'hui en sixième sans savoir lire. Elle implique également une pratique correcte par les élites de notre pays. Il existe encore des mordus de la langue, comme les candidats à la dictée de Bernard Pivot, mais des efforts sont indispensables pour rendre son prestige à la langue française. Celle-ci est un patrimoine que les députés et notamment ceux de la commission des affaires culturelles se doivent de défendre ". Voilà un langage bien dans les normes traditionnelles. Cependant le sondage de la SOFRES révèle que si la majorité des personnes interrogées pense que l'école est importante pour la défense de la langue française, un nombre important estime que les Français eux-mêmes doivent faire des efforts (il y avait plusieurs réponses possibles à la question « À qui faites-vous le plus confiance pour défendre la langue française?» et deux réponses étaient permises par personne interrogée : $59 \%$ ont donné l'école comme réponse, $29 \%$ les Français euxmêmes, $28 \%$ l'Académie, $15 \%$ les médias, $10 \%$ le gouvernement, $8 \%$ les autres pays francophones, $3 \%$ les entreprises). $38 \%$ ont même répondu que c'est le mauvais niveau d'enseignement du français à l'école qui menace le plus la langue française. L'école ne serait plus vue comme le seul instrument possible de défense de la langue. Il y a donc eu évolution dans ce domaine. 
18 La dernière phase dans la politique linguistique française correspond à une série de mesures prises depuis les années 1960, la plus frappante étant que, depuis 1992, le principe selon lequel «la langue de la République est le français» est inscrit dans la Constitution. Certains ont vu dans cette décision une politique de défense par rapport à l'emprise de l'anglais. En effet, le problème du recul de la langue française est apparu dès la fondation de l'Organisation des Nations unies en 1945: le français ne fut accepté comme langue de travail qu'à la suite d'un vote favorable obtenu à une seule voix de majorité, et en 1967 il fallut un débat spécial pour obtenir la publication des communications en français aussi bien qu'en anglais. C'est en fait l'arrivée dans les institutions et organismes internationaux de pays nouvellement indépendants et appartenant avant leur indépendance à l'empire colonial français qui a sauvé le français au niveau international. Les gouvernements français - mais pas la population française en sont très conscients, d'où leur engagement au sein des institutions de la francophonie, engagement discret jusqu'aux années 1980, de peur de se voir accusés de colonialisme, mais manifesté au grand jour depuis la création du Haut conseil de la francophonie en 1984 par M. Mitterrand, celui-ci ayant annoncé que la francophonie le "passionnait » et qu'il allait «s'en occuper ». Cet engagement s'est concrétisé de plus en plus au cours des dix dernières années. C'est ainsi que $M$. Legendre déclarait au Sénat, le 22 novembre 1993: "Grâce à la francophonie, la France conserve une dimension mondiale [...] en intervenant dans le débat sur le GATT pour soutenir à l'unanimité l'exigence de l'exception culturelle, les pays francophones ont fait preuve de détermination. Ils ont montré aussi que la francophonie n'est pas seulement un mouvement culturel, mais aussi une force politique qui compte » (notons d'ailleurs que le principe de l'exception culturelle au GATT a été précédé par l'exclusion des «industries culturelles » de l'ALENA - Accord de libre-échange nord-américain - en 1988).

Les gouvernements français, conscients donc dès l'après-guerre du nouveau danger auquel la langue devait faire face au niveau international, à savoir la domination de l'anglais, ont pris un certain nombre de mesures défensives à l'intérieur du pays. La première fut la création du Haut comité de défense et d'expansion de la langue française par le général de Gaulle en 1966 qui devint en 1973 le Haut comité de la langue française dont les buts n'étaient pas seulement de défendre la qualité du français et d'accroitre son influence à l'étranger mais aussi de coopérer avec les pays francophones; en 1984 celui-ci fut remplacé par deux organismes, le Comité consultatif de la langue française et le Commissariat général de la langue française qui allaient devenir en 1989 le Conseil supérieur de la langue française et la Délégation générale à la langue française dont les rôles étaient respectivement de discuter et promouvoir un nouveau «bon usage » et d'en disséminer les modalités en France et à l'étranger.

La loi Bas-Lauriol, qui établissait l'emploi obligatoire du français dans les domaines qui commençaient à se ressentir des incursions de l'anglais, fut présentée à l'Assemblée nationale en 1973, et elle fut votée à l'unanimité en 1975. Il s'agissait surtout des étiquetages (terme qui inclut l'étiquette sur un produit et les notices d'emploi, de garantie ou donnant des informations supplémentaires) et de la publicité, ceci pour la protection du consommateur ; au travail, il s'agissait de rendre le français obligatoire au niveau des directives données et surtout au niveau des contrats, pour protéger les employés. Cette loi établissait également que l'emploi des nouvelles terminologies officielles serait obligatoire dans tous les documents d'origine gouvernementale, dans les contrats, dans le domaine de l'éducation nationale et toutes les institutions de l'État. 
21 Qu'étaient ces terminologies officielles ? Dès le départ, ceux qui s'inquiétaient du sort de la langue française étaient convaincus que le problème était essentiellement d'ordre terminologique, le français empruntant trop à l'anglais, surtout dans certains domaines dans lesquels la France n'était pas initiatrice de progrès. D'où un premier décret en janvier 1970 établissant le besoin d'avoir des commissions de terminologie au sein des ministères. D'autres suivirent, spécifiant de manière plus précise la nature et le fonctionnement de ces commissions et de nombreux arrêtés furent publiés dans le Journal officiel, donnant des listes de termes choisis dans chaque domaine représenté par un ministère. Ces termes sont rassemblés de temps en temps dans des dictionnaires de termes officiels. Depuis 1990, ces commissions incluent également des représentants officiels de divers pays francophones.

Notons dans ce contexte deux graves problèmes. En premier lieu, ces commissions étant composées de volontaires, souvent des amateurs de langue plutôt que des linguistes, elles sont de valeur très inégale (ce qui explique carences et erreurs, quelquefois ridicules, voir le nouveau Dictionnaire des termes officiels de 1994). Ensuite, comment rendre exécutoires les décisions prises? Dans la loi Bas-Lauriol, la mise en vigueur des décisions prises dépendait de la direction de la consommation et de la répression des fraudes, de la direction générale de la concurrence et de la consommation et du service des douanes. Or aucune de ces directions n'a considéré la langue comme un domaine prioritaire; le résultat est que la loi n'a jamais été vraiment appliquée, d'où la nouvelle loi Toubon qui cherche non seulement à combler certaines lacunes du texte précédent mais à lui donner plus de poids. On peut cependant s'interroger sur ce dernier point à cause de la lenteur de la procédure. Le projet de loi Tasca, qui a précédé la loi Toubon, aurait été beaucoup plus efficace. En effet, l'article 9 prévoyait la consignation de tout produit ou document en infraction dans un local non accessible au public. Dans le projet Toubon, par contre, il n'y a pas la possibilité de consigner quoi que ce soit, ce qui signifie que le temps que la procédure soit faite, les produits et leur documentation litigieuse auront déjà été vendus ou redistribués. De plus les règles du Marché commun font que la loi ne peut protéger que le consommateur final, et non les intermédiaires dans la chaîne commerciale, ce qui a pour effet de grandement diminuer la portée de toute loi de ce genre.

Une des raisons qui explique les mesures défensives décrites ci-dessus est le surplus d'influence qu'a pris l'anglais depuis l'entrée de la Grande-Bretagne dans le Marché commun, ou du moins la perception qu'en ont beaucoup de Français. Il leur semble, en effet, que depuis cette époque le français est en perte de vitesse non seulement à l'échelle internationale mais même en Europe : c'est encore la première langue étrangère en Grande-Bretagne et en Irlande, mais sa position dans les pays latins et en Grèce est en voie d'effondrement. Tout élargissement de l'Union européenne est vu comme un facteur certain d'aggravation de la situation. Pourtant en 1972, le président Pompidou et le premier ministre Heath s'étaient entendus pour que les Britanniques jouant un rôle officiel à la Commission soient capables de travailler en français. La France avait prévu à ce moment-là un programme d'enseignement combinant cours de langue et stages dans le pays. Or les résultats sont perçus comme peu satisfaisants (ce n'est pas l'opinion des Anglais travaillant à Bruxelles qui sont tous appelés à employer le français). Actuellement, un programme de formation en français pour les fonctionnaires autrichiens et Scandinaves appelés au siège de la Commission a été mis en place, mais quelles chances a-t-il de réussir ? Selon Xavier Deniau, exprimant l'avis de la commission des affaires étrangères sur le projet de loi Toubon ${ }^{3}$, non seulement l'expérience 
précédente est peu rassurante mais, de plus, les moyens seraient insuffisants. La meilleure voie pour lutter contre le "tout anglais", selon Deniau et de nombreuses autres personnalités, serait de jouer « la carte du plurilinguisme» qui recommande que plusieurs langues soient enseignées dans chaque pays membre. La France a l'intention de faire des propositions allant dans ce sens en 1995 lorsqu'elle assurera la présidence de l'Union européenne. Elle espère être soutenue particulièrement par l'Allemagne et l'Autriche, ainsi que par les autres pays non anglophones qui sont confrontés à des problèmes semblables (sauf les pays Scandinaves qui pratiquent l'anglais).

Mais si le français est en recul ce n'est certainement pas à cause de la politique linguistique de la Communauté européenne, puisque celle-ci, en principe, n'en a pas. Bien au contraire. En effet le Marché commun est né de l'extension de la CECA, dominée au départ par trois pays de langue française, la France, la Belgique et le Luxembourg, d'où, en fait, une prépondérance du français dans la Communauté européenne. Ainsi, la seule version officielle du texte mettant en place la CECA est la version française. C'est encore la seule langue de la Cour de justice de la Communauté européenne à Luxembourg dans la mesure où les juges y délibèrent en secret en français, sans qu'aucun traducteur n'y ait accès et les jugements sont prononcés en français (il va de soi que les plaidoiries sont dans la langue des plaideurs). C'est également la langue de communication la plus employée au sein des services de la Commission européenne, au point que les journalistes de langue anglaise se plaignent de ce que les conférences de presse sont toujours en français; de plus, si l'on téléphone à la Commission, il vaut mieux le faire en français car les secrétaires sont dans l'ensemble francophones. Tous les projets de directives sont rédigés d'abord en français. Les directions générales travaillent également en français. Cependant il est vrai que depuis l'entrée de la Grande-Bretagne dans le Marché commun l'emploi de l'anglais a beaucoup progressé, en partie à cause de son rôle de langue internationale, non seulement dans le monde politique, mais également dans celui des finances, de l'économie et de la recherche. C'est ainsi qu'au cours de ces dernières années le personnel des directions générales est devenu beaucoup plus bilingue et que dans certaines sphères l'anglais progresse d'une manière alarmante pour le français. Ceci concerne particulièrement les domaines de la recherche, des relations extérieures en matière d'économie et de la concurrence à cause des contacts avec les multinationales. La Communauté semble donc avoir été un rempart de défense et d'illustration du français dans le passé, et par là même une source de puissance, ne serait-ce que morale. Mais c'est là une position que le français est appelé à partager de plus en plus avec l'anglais et, peutêtre, bientôt avec l'allemand qui commence à vouloir s'y affirmer (ceci n'est guère surprenant lorsque l'on considère le fait que la population de l'Allemagne ajoutée à celle de l'Autriche représente une proportion importante de la population de l'Union européenne et qu'elle contribue plus que tout autre pays aux finances de cette dernière).

Ce qui inquiète le plus certains Français est que la compétence des États à légiférer en matière linguistique ne leur semble pas automatiquement acquise au sein de la Communautét. La raison en est que certaines législations nationales peuvent être contraires au principe de la libre circulation des marchandises et des services prescrit par les articles 30 et 59 du traité de Rome. C'est ainsi qu'il y a eu des cas de pays qui ont cherché à tricher en demandant des traductions officielles et conformes pour des étiquetages fort simples, tout simplement pour retarder l'entrée de produits étrangers dans leur pays (voir l'affaire 154/87/CEE contre l'Italie). Il y a eu aussi des pays - la France, la Grèce et l'Italie - qui ont essayé de limiter l'exercice de la profession de guide 
touristique aux citoyens de ces pays. La Cour de justice des Communautés européennes s'est prononcée contre de telles pratiques contraires à l'esprit du traité de Rome (voir les trois jugements du 26 février 1991 : les affaires 154/89/CEE, 189/89/CEE et 198/89/CEE). C'est également au nom du traité de Rome que fut amorcée une procédure précontentieuse contre la loi Bas-Lauriol mais celle-ci ne fut pas menée à son terme. Cependant la France dut à cause de cela écarter du champ d'application de la loi les échanges de marchandises entre professionnels (décision administrative du 13 avril 1983 modifiant la circulaire d'application du 30 décembre 1982). En d'autres termes, la loi BasLauriol et la nouvelle loi Toubon ne peuvent défendre que le consommateur final, et non les intermédiaires comme les producteurs et les grossistes.

Le résultat de l'apparition de ce genre de problème est que petit à petit la question linguistique a été abordée dans certaines directives, mais de manières fort différentes, d'où inquiétudes et malentendus. C'est ainsi que la directive 79/112/CEE sur l'étiquetage des denrées alimentaires prévoit le recours «à une langue facilement compréhensible pour les acheteurs » alors que d'autres favorisent les langues nationales.

L'expression «langue facilement compréhensible pour les acheteurs » a alarmé certains Français estimant qu'il s'agit d'un concept difficile à définir et aisément contournable, susceptible de conduire au " tout anglais » tant redouté. Or les intentions des législateurs étaient tout autres puisque cet aspect de la directive s'adressait surtout aux pays ayant plusieurs langues officielles comme la Belgique ou l'Espagne, pour éviter que tout n'y soit traduit en plusieurs langues lorsque cela n'était pas strictement nécessaire à la compréhension du consommateur. N'ont donc pas besoin d'être traduits: les mots étrangers n'ayant pas d'équivalent dans la ou les langues officielles de l'État membre où le produit est importé ; des mots dont l'orthographe ne varie que légèrement d'une langue à l'autre; des expressions connues comme made in. Notons que le jugement du 7 mars 1990 de la Cour de justice des Communautés européennes a maintenu que « le besoin de bien informer le consommateur est absolument prioritaire dans le cadre de la CE» (affaire 362/88/CEE), ce qui implique dans tous les autres cas l'emploi des langues maternelles ou, à défaut, des langues nationales et officielles. Le champ d'application de la directive 79/112/CEE, qui permet un étiquetage multilingue (article 14), a été d'ailleurs élargi par le jugement de la Cour de justice des Communautés européennes du 18 juin 1991, dit « arrêt Peeters » (affaire 369/89/CEE), qui a estimé qu' « un État membre ne pouvait imposer l'utilisation exclusive de sa langue nationale ou d'une de ses langues régionales sans retenir la possibilité que soit utilisée une autre langue ", car ceci irait audelà des intentions de l'article 30 du traité de Rome.

Malgré la nature non agressive prise par la CE à l'égard des différents pays membres, nombre d'entre eux se sentent menacés du point de vue de leur intégrité linguistique; presque tous ont maintenant une législation prescrivant l'emploi de leur(s) langue(s) officielle(s) dans certains contextes précis. Pour ce qui est de la protection du consommateur, par exemple, c'est seulement en Allemagne, en Irlande, au Luxembourg et en Grande-Bretagne que le problème de la protection du consommateur du point de vue linguistique n'a été discuté ni au niveau des industries concernées, ni au niveau des associations de consommateurs. La Belgique, l'Espagne, l'Italie, la Grèce et le Portugal ont tous adopté des législations visant la protection de leurs consommateurs à ce niveau.

Il y a donc eu une certaine confusion chez certains quant à l'attitude de la CE en matière linguistique. C'est pour cela que le Conseil de l'Europe a demandé à la Commission d'étudier le problème de plus près, spécialement pour ce qui est des étiquetages, et de 
présenter des recommandations au Conseil et au Parlement européen. Celles-ci ont été publiées le 10 novembre 1993 [COM (93) 456]. Ce document examine les différents genres de recommandations linguistiques existant dans les textes de droit européen, les différentes approches adoptées par les États membres, la nature des problèmes linguistiques attestés et propose des suggestions quant à la manière de standardiser l'approche communautaire dans ce domaine.

Le principe directeur est que la langue ne doit pas gêner la libre circulation des marchandises et des services à l'intérieur de la $\mathrm{CE}$ et que le consommateur doit toujours être protégé. Que cela signifie-t-il en pratique au niveau des marchandises? Que chaque État membre a le droit d'interdire l'entrée de marchandises mal étiquetées selon lui sur son territoire si elles viennent d'un pays ne faisant pas partie de l'Union européenne. Des marchandises venant du Japon, par exemple, accompagnées d'une notice anglaise peuvent enfreindre la loi française. Cependant si un pays de l'Union européenne, l'Espagne par exemple, accepte d'importer des produits avec des notices en anglais et les revendre en France à des grossistes, la France ne peut pas en interdire l'entrée si l'importation était conforme aux termes de l'article 10 du traité de la Communauté européenne. C'est ce que l'on nomme en France être en « libre pratique » par opposition à la "libre circulation" (notons que la langue anglaise ne contient pas de mot pour exprimer cette opposition, le terme free circulation étant employé dans les deux cas). Tout produit originaire de la Communauté bénéficie bien entendu du régime de libre circulation à l'intérieur de celle-ci. Cependant, que les produits soient en libre pratique ou en libre circulation, il y a des règles linguistiques à observer quant à leur étiquetage dans certains cas précis.

31 Au niveau des travailleurs, cela signifie que l'on n'a pas le droit de discriminer contre des travailleurs pour des questions de langue, sauf si celle-ci est essentielle pour le travail à accomplir. Pour ce qui est des contrats, il est considéré normal qu'une entreprise française employant des travailleurs essentiellement français rédige ses contrats de travail en français; cependant s'il s'agissait d'une entreprise anglaise en France employant des employés anglais, il serait possible de rédiger les contrats en anglais ou en français. L'arrêt Groener stipule également qu'il est possible d'exiger un certain niveau de compétence linguistique pour certains emplois.

De toute évidence l'approche adoptée en matière linguistique est quelque peu anarchique. Elle a besoin d'être standardisée et rendue plus systématique. C'est ce que conclut le rapport de la Commission du 10 novembre 1993. Ainsi la France, en adressant à la Commission ses plaintes et ses craintes, reflète une inquiétude générale et pas seulement une obsession nationale. Il y a de toute évidence des problèmes à régler. Pour cela, il faut tenir compte des différentes politiques linguistiques adoptées par les États membres et de l'équilibre à maintenir entre la protection du consommateur et le principe du libre échange. La Commission a suggéré en particulier de développer le plurilinguisme, d'aborder de manière plus systématique les questions linguistiques lors de l'élaboration de textes législatifs européens et au besoin de réviser certaines directives afin d'y incorporer un élément linguistique. Un autre problème à aborder est la détermination de la responsabilité pour les traductions ou documents en langue(s) nationale(s) du pays où doit être vendue la marchandise. C'est en principe la responsabilité du producteur, mais il peut y avoir des désaccords dans des cas particuliers.

Pour récapituler, du point de vue de la France, les changements institutionnels décrits cidessus reflètent une prise en considération de la dimension francophone et la mise en 
place d'une politique beaucoup plus agressive en matière de défense du patrimoine linguistique. Cependant ces changements illustrent dans l'ensemble une attitude défensive plutôt qu'illustrative. Certains accusent la France de mal accepter la décolonisation et le fait de ne plus être une grande puissance mondiale; ceux-là sont en général très négatifs au sujet de sa politique linguistique actuelle. D'autres pensent qu'il s'agit du principe du droit à sa langue; ils montrent par conséquent quelques remords quant au traitement passé des langues régionales, ils se plongent dans la défense du plurilinguisme et, au nom du monde francophone qui « compte sur nous ", ils proclament le besoin de défendre la bonne évolution du français. Toutefois, il ne s'agit plus, en général, de " chasse » aux mots, comme le prétendent les commentateurs hostiles à toute politique en matière linguistique, mais de canaliser les développements de la langue française en France et dans le monde.

Du point de vue de la Communauté européenne, il en va tout autrement. Il semble qu'au départ personne ne se soit vraiment inquiété du rôle des langues en présence, d'où, à l'heure actuelle, un certain nombre de problèmes qui proviennent essentiellement d'un manque de standardisation systématique des règlements en matière linguistique. Des efforts semblent être faits pour y remédier. Pour ce qui est des langues nationales employées dans leur pays, le principe de la subsidiarité, qui semble bien établi maintenant, devrait en principe les protéger toutes. Mais au niveau des institutions centrales, le plurilinguisme est trop cher et trop long. On peut donc s'attendre à voir s'établir deux ou trois langues de travail : l'anglais et le français sont déjà en place et il est possible que vienne s'y ajouter l'allemand.

\section{NOTES}

1. Sondage SOFRES des 4 et 5 mars 1994 auprès d'un échantillon national représentatif de 1000 personnes (NDLR).

2. Les sociolinguistes britanniques sont souvent sévères à l'égard de cette politique visant le monolinguisme, de manière injuste, car là où ils ne voient que totalitarisme, il y avait un vrai désir de justice sociale. De plus la Grande-Bretagne est arrivée au même degré de monolinguisme ou presque, en employant des moyens beaucoup plus détournés et moins avouables. Cependant le côté monolithique de la situation linguistique française pose aujourd'hui des problèmes d'adaptation pour les immigrés et les personnes tenant à leur héritage culturel. Il y a en fait pour ces dernières un problème au niveau des principes : si pour avoir accès au principe d'égalité il faut que tous parlent une langue nationale, qu'advient-il du principe de liberté quand celui-ci signifie le droit de parler une langue maternelle autre que le français? Liberté et égalité linguistiques semblent contradictoires dans la réalité, et c'est le principe d'égalité qui semble avoir dominé en France : une des raisons pour lesquelles les langues régionales sont en déclin est que peu de parents sont prêts à ce que leurs enfants apprennent une langue régionale à la place de l'anglais ou d'une autre matière "utile », justement au nom de l'égalité des chances. C'est ce même phénomène qui met de très nombreuses langues dans le monde en danger de disparition à l'heure actuelle.

3. Rapport présenté à l'Assemblée nationale le 28 avril 1994. 
4. Voir le rapport de M. Legendre sur le Sommet de l'île Maurice présenté au Sénat le 22 novembre 1993 et celui de M. Deniau présenté le 28 avril 1994 à l'Assemblée nationale au nom de la commission des affaires étrangères sur le projet de loi Toubon.

\section{RÉSUMÉS}

D'un point de vue anglo-saxon, la politique défensive menée par la France pour protéger son patrimoine linguistique s'inscrit d'abord dans la longue durée d'une tradition de planification étatique, mais, aujourd'hui, elle doit composer avec ces deux impératifs européens que sont la défense du consommateur et le libre-échange.

\section{INDEX}

Index géographique : France

Mots-clés : communauté européenne, politique linguistique, langue régionale, planification linguistique

\section{AUTEUR}

\section{ANNE JUDGE}

Université du Surrey 